\title{
CALIDAD DE VIDA, SENTIDO DE COHERENCIA Y NIVELES DE SEDENTARISMO EN ACADÉMICOS (AS) Y ADMINISTRATIVOS (AS) DEL CAMPUS PRESBÍTERO BENJAMÍN NÚÑEZ, UNA
}

\author{
Pedro Ureña Bonilla \\ Doctor en Ciencias del Deporte con especialidad en \\ Psicología Aplica da al Ejercicio \\ purena@medvet.una.ac.cr
}

RESUMEN

\begin{abstract}
El propósito de este estudio fue analizar la Calidad de Vida de académicos (as) y administrativos (as) del Campus Presbitero Benjamín Núñez de la Universidad Nacional, Costa Rica. Asimismo, valorar la influencia de los factores Sentido de Coherencia y Ejercicio Físico en la Calidad de Vida de los supracitados trabajadores (as). En el estudio participó un grupo de 37 académicos (as) y 30 administrativos (as). Se aplicó el cuestionario de Calidad de Vida SF-36, la escala de Sentido de Coherencia y un cuestionario para medir hábitos relacionados con el Ejercicio Físico. Los resultados encontrados mostraron en general valores correspondientes a una Calidad de Vida relativamente buena. Similar fue la valoración que se hizo en cuanto al Sentido de Coherencia. Asimismo, se encontró relaciones significativas entre algunos aspectos del Sentido de Coherencia y de la Calidad de Vida. También, se evidenció que las personas que realizan Ejercicio Físico, comparadas con las que no lo hacen, poseen una mejor Calidad de Vida. Se concluyó de manera general que, aunque la Calidad de Vida y el Sentido de Coherencia fueron relativamente buenos, es necesario diseñar y ejecutar propuestas con el propósito de mejorar ambos aspectos. En este sentido, el Ejercicio Físico se delineó como uno de los pilares a partir de los cuales se podría ejecutar propuestas innovadoras para la salud de los (as) trabajadores (as) universitarios(as), todo ello, en el marco de lo que se denomina "Universidades Saludables"..
\end{abstract}

PALABRAS ClAVES: Calidad de Vida, Sentido de Coherencia, Ejercicio Físico.

\section{QUALITY OF LIFE, SENSE OF COHERENCE AND LEVELS OF SEDENTARISM OF FACULTY AND STAFF FROM THE PRESBÍTERO BENJAMÍN NÚÑEZ CAMPUS, UNA}

\section{ABSTRACT}

The aim of this study was to analyze the quality of life of faculty and staff of the Presbitero Benjamin Núñez Campus of Universidad Nacional (UNA, Costa Rica) as well as to assess the influence of two factors, sense of coherence and physical exercise, in the quality of life of the subjects. A group of 37 faculty members and 30 staff members participated in the study. The SF-36 Questionnaire, the Sense of Coherence Scale, and a survey to measure physical exercise habits were used. In general, results showed a relatively good quality of life and similar scores were found in the Sense of Coherence Scale. Additionally, significant connections were found between certain factors related to sense of coherence and quality of life. Data confirmed that persons who practice physical exercise have a better quality of life than those who are not physically active. It was concluded that, although quality of life and sense of coherence were relatively good, new proposals must be designed and implemented to improve both aspects. In this sense, physical exercise proved to be one of the pillars in the development of innovative proposals aimed at bettering health in university employees, all of this within the "Healthy Universities" concept.

KEY WORDS: Quality of life, sense of coherence, physical exercise. 


\section{MHSalud}

URL www.una.ac.cr/mhsalud

\section{INTRODUCCIÓN}

En el contexto de lo que Peterken y otros (1996) denominan "Universidades Saludables", el estudio sobre la calidad de vida de quienes conviven en estos centros académicos es fundamental para la construcción pertinente de modelos de promoción de la salud (Antonovsky, 1996).

El concepto Calidad de vida es multidimensional (Simón, 1999) y ha estado ligado históricamente a constructos como felicidad y bienestar (Nordenfeld, 1993; Diener, 1994). Sin embargo, no se cuenta aun con una definición consensuada (Simón, 1999). Por ejemplo, Levi y Anderson (1980) entienden la calidad de vida como un constructo formado por las dimensiones bienestar físico, mental y social. Dicho nivel de bienestar está determinado por la forma en que cada persona lo perciba; es decir, es de carácter subjetivo. La felicidad, la satisfacción y la recompensa son consecuencias del nivel de bienestar de las personas. De manera que, todo lo que produzca sensación de bienestar incide positivamente en la calidad de vida.

En sentido similar, Brengelmann (1986) asocia la calidad de vida de las personas a situaciones específicas como: disfrutar de libertad, cultivar la relaciones sociales, tener la posibilidad de desarrollar la propia iniciativa, estar satisfecho, presencia reducida de problemas psicosomáticos, tener una buena profesión, un buen empleo y finalmente, encontrarle sentido a la vida. En consecuencia, si se cumple una o varias de las condiciones mencionadas, y además se juzgan positivamente, podría hablarse de una satisfacción vital favorable (Veenhoven, 1991), lo que sin lugar a duda, favorece la salud y la calidad de vida.

De manera resumida, Simón (1999) afirma que cualquier definición de calidad de vida debe considerar: las capacidades cognoscitivas, conductuales y de bienestar emocional. Asimismo, el sentimiento subjetivo de bienestar y satisfacción vital. También, las condiciones socio materiales objetivas de existencia y la percepción subjetiva de las mismas. Por otro lado, es preciso incorporar la satisfacción derivada de las condiciones materiales reales y concretas, así como psicológicas de las personas. Complementariamente, debe valorarse la percepción subjetiva del nivel de salud personal y su capacidad para comportarse de una forma subjetivamente satisfactoria. Finalmente, debe incorporar la percepción de la utilidad del significado de vivir y la satisfacción de las necesidades básicas del ser humano.

Efectivamente, se trata de un constructo integral compuesto por factores somáticos, psicológicos, sociales y culturales, donde no solo juega un papel determinante la dimensión objetiva, sino y de manera particular, la subjetividad de las personas.

Sin lugar a duda, la calidad de vida está íntimamente relacionada con la salud (Sánchez, 1996). De manera que, al promocionar una mejor calidad de vida, se está promoviendo una mejor salud. En este contexto, se entiende la salud, como un proceso de relaciones dinámicas y bidireccionales entre dimensiones y competencias individuales (biopsicosociales) y características ambientales (biofísicas, sanitarias, socioeconómicoculturales) cuyo resultado es un estado caracterizado por el equilibrio y el correspondiente bienestar psicosocial (Godoy, 1999, p. 43).

Con este marco de referencia y enfocando la atención en la dimensión de las competencias psicológicas, el Sentido de Coherencia (Antonowsky, 1987) puede entenderse como un factor potenciador de la salud y en consecuencia de la calidad de vida (Ebert, Tucker \& Roth, 2002; Lustig, Rosenthal, Strausser \& Haynes, 2000, Strausser \& Lustig, 2003). De acuerdo con Godoy (1999), el sentido de coherencia se constituye en un factor protector de la salud, o bien, en un recurso de resistencia o competencia. 


\section{MHSalud}

URL www.una.ac.cr/mhsalud

El sentido de coherencia refleja una posición básica ante la vida, una visión de mundo particular, una visión de vida ante acontecimientos inesperados y ante la vida misma (Antonowsky, 1987). De igual forma que las experiencias le pueden dar solidez al sentido de coherencia, también caracterizan la forma en que las personas construyen sus experiencias. Esa forma de ver las cosas y de relacionarse con ellas se desarrolla durante los primeros 10 años de vida y se consolidan a más tardar a los 30 años (Antonowsky, 1997). Después el sentido de coherencia permanece hasta el fin de la vida sin cambios importantes. Se trata de una orientación disposicional en relación con las cosas, personas y acontecimientos de la vida, donde asume una función directriz. El sentido de coherencia es una orientación general que se manifiesta como un sentimiento dinámico de confianza, de manera que los estímulos internos o externos son estructurados, predecibles y explicables. Por otro lado, se poseen los recursos para hacerle frente a las exigencias que representan esos estímulos y finalmente, los estímulos son vistos y valorados como algo que vale la pena enfrentar y superar (Antonowsky, 1997). Un sentido de coherencia fuertemente consolidado es base fundamental para un patrón de reacciones y acciones flexible y adaptativo (Antonowsky, 1997).

Antonowsky (1997) se refiere a tres factores fundamentales relacionados con el sentido de coherencia: La Comprensibilidad (componente cognoscitivo), la que se refiere a la medida en que una persona ordena, predice y explica estímulos internos y externos. Es decir, se trata de un aspecto cognitivo relacionado con el patrón de procesamiento de información. Con base en Moreno, González y Garrosa (1999) altos puntajes en este factor se asocian con una mayor capacidad para interpretar los estímulos del medio de manera no amenazante, aspecto que juega un papel central en la vivencia del estrés y consecuentemente en la calidad de vida de las personas. La Manejabilidad (componente instrumental), describe la confianza instrumental de la persona, su convicción de poder superar las situaciones de la vida presente o futura con las propias fuerzas o con ayuda de los demás. Finalmente, la Significatividad (componente motivacional), tiene que ver con el sentido de la propia vida y se le considera el aspecto motivacional emotivo del sentido de coherencia. Representa el grado de compromiso de la persona en diferentes áreas de la vida. Esta dimensión denota una inversión emocional en la vida y tiene que ver con la visión del sujeto de ciertas áreas de ésta como dignas de invertir tiempo y energía. Puntuaciones altas en esta dimensión son evidencia de que difícilmente la vida será vista como poco provechosa e insignificante, contribuyendo de manera importante al proyecto de vida de las personas (Moreno y otros, 1999).

La relación entre sentido de coherencia y salud es vista por Antonowsky (1997), de la siguiente manera: el sentido de coherencia puede ejercer influencia directa sobre el cerebro, el sistema inmunológico y el sistema hormonal de las personas, desencadenando reacciones distintas en diferentes niveles, de manera que, puede incidir en forma reguladora cuando presentan estados de tensión, o como filtro directo en el procesamiento de información. Asimismo, el sentido de coherencia puede actuar de manera directa en el procesamiento del estrés, en la medida en que moviliza recursos que contribuyen con la reducción de la tensión (Lundquist, 1995; Johansson et al; Poppius, et al, 1999). Finalmente, las personas que disponen de un sentido de coherencia fuerte, están más en condición de asumir comportamientos saludables, por ejemplo, una alimentación sana, toman previsiones y evitan comportamientos no saludables.

En estudios realizados por Palsson, Hallberg, Norberg y Byoervell (1996) se encontró una relación inversa entre los niveles de sentido de coherencia y la sensación de agotamiento crónico (burn out) producida por el trabajo. Es decir, entre más fuerte sea el sentido de coherencia, menor será la sensación de agotamiento emocional y despersonalización asociados con la vivencia de estrés crónico (Baker, North y Smith, 1997, Gilbar, 1998). En este mismo sentido, los trabajos reportados 


\section{MHSalud}

URL www.una.ac.cr/mhsalud

por Carmel, Anson, Levenson, Bonneh y Maoz (1991), Flannery y Flannery (1990), Moreno, Alonso y Alvarez (1997), Moreno González y Garrosa (1999) y Ryland y Greenfeld (1991) destacan el rol relevante que juega el sentido de coherencia como variable mediadora en los procesos de salud (Lustig, 2005).

En relación con la promoción de la calidad de vida se puede utilizar diversos enfoques. Por ejemplo Evans (2000) agrupa dos estrategias básicas: programas dirigidos a las personas y programas dirigidos al ambiente con que interactúan las personas. Asimismo, Halpern (1994) y Schalock (1994) sugieren trabajar en tres dominios específicos: vida en la comunidad y en el hogar, empleo y funcionamiento de salud. Otros autores como Rommey, Brown y Fry (1994) subrayan la importancia de realizar todo tipo de actividades que incrementen la felicidad en las personas. De manera particular se refieren a la relevancia de promover el desarrollo de la autoeficacia, la autoestima y de mecanismos de afrontamiento de estrés y solución de problemas. Otro de los factores fundamentales en la promoción de la salud corresponde al apoyo social (Fernández y García, 1999). Este es definido como el acceso y la utilización de personas y organizaciones para afrontar acontecimientos, para los cuales la persona no posee un comportamiento adaptativo inmediato (Gracia, Herrero y Musito, 1995; Robinson y Garber, 1995).

En esencia se trata de promover patrones de comportamiento que favorezcan una mejor calidad de vida y consecuentemente una mejor salud. El ejercicio físico es una de actividades cuyo impacto integral puede convertirse en una estrategia fundamental en la promoción de la calidad de vida y de la salud (Sánchez, 1996). El ejercicio físico, controlado correctamente en su frecuencia e intensidad, se asocia con efectos positivos en aspectos como: la condición cardiovascular, la composición corporal y obesidad, la presión sanguínea y los niveles de colesterol (Sánchez, 1996). También el ejercicio físico diseñado apropiadamente en cuanto a su dinámica socio - afectiva, puede asociarse con la salud psicológica (sensación de bienestar o satisfacción con la vida, estado anímico y ansiedad) (Ureña, 2005; Hollmann y Struder, 2001; Weinberg y Gould, 1999).

Hay una serie de mecanismos que se suele señalar como responsables de los beneficios que tiene el ejercicio físico en la salud psicológica (Weyerer y Kupfer, 1994). Los de carácter biológico destacan cinco procesos: 1) el incremento en la temperatura corporal, el cual tiene un efecto tranquilizante integral, 2) el ejercicio facilita el afrontamiento del estrés, al incrementar la actividad adrenal, lo que hace que las reservas de esteroides disponibles aumenten, 3) el ejercicio físico reduce el potencial nervioso del músculo en reposo lo que ocasiona una liberación de la tensión, 4) el ejercicio físico facilita la neurotransmisión de la norepinefrina, la serotonina y la dopamina, lo que provoca una mejoría del estado anímico y 5) el ejercicio físico según intensidad, promueve la liberación de endorfinas, lo que produce bienestar psicológico (Plante y Rodin, 1990)

Entre los mecanismos de carácter psicológico, se señalan: 1) la mejor condición física producto del ejercicio físico brinda sensación de competencia, control y autosuficiencia, 2) el ejercicio es una forma de meditación que desencadena estados de conciencia alterados, induciendo relajación, 3) el ejercicio es una forma de retroalimentación que enseña a las personas a regular su propia activación, 4) el ejercicio físico proporciona distracción, diversión, o tiempo de evasión de pensamientos, emociones y conductas desagradables, 5) el ejercicio físico estimula el contacto entre las personas lo que favorece el refuerzo social, facilitándose así estados psicológicos positivos y 6) el ejercicio físico compite con estados negativos como la ansiedad y la depresión a nivel de sistema cognitivo y somático.

Congruente con la explicación de mecanismos expuesta antes, el Instituto Nacional de la Salud Mental de los Estados Unidos (Morgan y Goldston, 1987), señala que el ejercicio físico incide 


\section{MHSalud}

URL www.una.ac.cr/mhsalud

positivamente sobre la salud mental en la medida en que, produce la sensación de bienestar, induce la reducción de emociones estresantes como la ansiedad, reduce estados depresivos (en caso de depresiones leves y moderados), ayuda a lograr estabilidad emocional y reduce la presencia de hormonas estrés (Weinberg y Gould, 1999; Stephens, 1988; McTeer y Curtis, 1993, LaForge, 1995; Steptoe, Moses, Edwards y Mathews, 1993; Weyerer y Kupfer, 1994).

Desde el punto de vista social, tal y como lo apunta (Sánchez, 1996), el nivel de operatividad motriz de una persona tiene repercusiones significativas en las posibilidades de interacción física con el entorno social, y consecuentemente en las posibilidades de las personas para comunicarse y relacionarse con otros. La dinámica del ejercicio físico según modalidad, facilita el establecimiento de interacciones, lo que podría tener efectos positivos de carácter psicosocial. En este sentido podría plantearse a manera de hipótesis que, con la práctica del ejercicio físico se relacionan, no solo la capacidad de rendimiento físico, sino también, la propia estima corporal, el afán de superación, la capacidad de interacción social, cooperación y del trabajo en equipo; amén de una serie de valores y actitudes (superación, voluntad, autocontrol, disciplina, sociabilidad, socioempatía) que se asocian con una buena calidad de vida (Weinberg y Gould, 1999; Comellas y Mercader, 1992).

De acuerdo con la revisión de literatura realizada, todo parece indicar que la calidad de vida está íntimamente relacionada con la salud y que el factor sentido de coherencia, juegan un papel central en la calidad de vida. Finalmente, también es válido afirmar que la dinámica somato-psíquica y social que tienen lugar durante el ejercicio físico, puede incidir positivamente en la calidad de vida de las personas y consecuentemente en su salud.

Con base en las afirmaciones precedentes, la presente investigación tuvo como propósito general, analizar la calidad de vida de los académicos (as) y administrativos (as) del Campus Presbítero Benjamín Núñez. Asimismo, valorar la influencia de los factores Sentido de Coherencia y Ejercicio físico sobre la calidad de vida de los trabajadores (as) del supracitado Campus de la Universidad Nacional.

\section{METODOLOGÍA}

Se trató de un estudio descriptivo correlativo en el que se describe cuál es la calidad de vida de los académicos (as) y administrativos (as) que laboran en el Campus Benjamín Núñez, de la Universidad Nacional. En este sentido, se midió una serie de dimensiones relacionadas con la calidad de vida que permitieron describir con precisión, cuál es la percepción subjetiva de la salud que tienen los supramencionados profesionales y la relación con el Sentido de Coherencia y sus hábitos asociados con el ejercicio físico.

Sujetos: Se contó con la participación de 30 administrativos (as) y 37 académicos (as). La edad promedio general fue de 36,7 años. El $40.6 \%$ trabaja en condición de interino (a) y el 59,4\% en condición de propietario (a). En términos promedio, el grupo tiene un tiempo de 9 años de laborar en la Universidad Nacional. La distribución de participantes por instancia fue la siguiente: El Centro Internacional de Política Económica 16, el CINAT 12, la Escuela de Medicina Veterinaria 15 y la Escuela Ciencias del Deporte 24.

Instrumentos: La información requerida en este estudio se recopiló con base en el Cuestionario de Salud (SF-36) (Ware y Sherbourne, 1992), la Escala de Sentido de Coherencia (Antonowsky, 1987) y un Cuestionario sobre hábitos relacionados con el ejercicio físico. 


\section{MHSalud}

URL www.una.ac.cr/mhsalud

\section{El cuestionario de salud (SF-36)}

Se trata de una escala genérica que proporciona un Perfil de la Calidad de Vida Relacionada con la Salud (CVRS) y aplicable tanto a la población en general como a pacientes (Vilagut et ál., 2005). El cuestionario está compuesto por 36 ítems que valoran los estados tanto positivos como negativos de la salud. Los 36 ítems cubren las escalas: Función física, compuesto por los ítems (3, 4, 5, 6, 7, 8, 9, 10, $11,12)$. Esta dimensión se refiere al grado en el que la salud limita las actividades físicas tales como el autocuidado, caminar, subir escaleras, inclinarse, coger y llevar cargas o pesos, y los esfuerzos moderados e intensos. Rol físico, integrado por los ítems $(13,14,15,16)$. Se trata del grado en el que la salud física interfiere con el trabajo y en otras actividades diarias, incluyendo rendimiento menor que el deseado, limitación en el tipo de actividades realizadas o dificultad en la realización de actividades. Dolor corporal, conformada por los ítems $(21,22)$. Tiene que ver con la intensidad del dolor y su efecto en el trabajo habitual, tanto fuera de casa como en el hogar. Salud general, integrada por los ítems (1, $33,34,35,36)$. Valoración personal de la salud que incluye la salud actual, las perspectivas de salud en el futuro y la resistencia a enfermar. Vitalidad, compuesta por los ítems $(23,27,29,31)$. Se refiere al sentimiento de energía y vitalidad, frente al sentimiento de cansancio y agotamiento. Función social, incluye los ítems $(32,20)$. Grado en el que los problemas de salud física o emocional interfieren en la vida social habitual. Rol emocional, ítems (17, 18, 19): Grado en el que los problemas emocionales interfieren en el trabajo u otras actividades diarias, incluyendo reducción en el tiempo dedicado a esas actividades, rendimiento menor que el deseado y disminución del esmero en el trabajo. Salud mental, ítems $(24,25,26,28,30)$ : Salud mental general, incluyendo depresión, ansiedad, control de la conducta y bienestar general.

Cada ítem se puntúa de 0 a 100, donde 0 es el peor estado de salud y 100 el mejor. El puntaje correspondiente a cada dimensión se suma luego de la conversión y se divide entre el número de ítems para obtener el promedio. El máximo total de puntuación alcanzable es 800, lo que indicaría una valoración subjetiva positiva y máxima de la salud.

El instrumento incluye un ítem de transición que pregunta por el cambio en el estado de salud general respecto al año anterior, sin embargo, ese ítem no se utiliza para el cálculo de ninguna de las escalas, pero proporciona información valiosa sobre el cambio percibido en el estado de salud. Se reporta una consistencia interna superior a 0,7 (alfa de Cronbach) en la mayoría de las dimensiones (rango de 0,71 0,94), excepto en la dimensión de relación social (alfa $=0,45)$. Los coeficientes de correlación intraclase, oscilan entre 0,58 y 0,99 (Alonso, Prieto y Antó, 1995).

\section{Escala Sentido de Coherencia}

Instrumento constituido por 29 ítems agrupados en tres sub-escalas que evalúan cada una de la tres dimensiones del constructo "Sentido de Coherencia": Comprensibilidad (describe la medida en que una persona ordena, predice y explica estímulos internos y externos. Se trata de un aspecto cognitivo relacionado con el patrón de procesamiento de información). Manejabilidad (describe la confianza instrumental de la persona, su convicción de poder superar las situaciones de la vida presente o futura con las propias fuerzas o con ayuda de los demás. Antonowsky (1997) define esa convicción como un patrón de procesamiento de información cognitivo - emocional) y Significatividad (se refiere al sentido de la propia vida y se le considera el aspecto emotivo - motivacional del Sentido de Coherencia (Antonowsky, 1997). La escala de respuesta presenta 7 categorías (de 1 a 7 ) que forman un continuo bipolar limitado en los extremos por dos frases de referencia a partir de las cuales se debe situar el sujeto. Las puntuaciones expresan la "fortaleza" del Sentido de Coherencia, siendo ésta 


\section{MHSalud}

URL www.una.ac.cr/mhsalud

mayor cuánto mayor sea la puntuación obtenida. La versión utilizada es la adaptada por Moreno Jiménez y otros (1997). Para efectos de análisis estadísticos, se trabajó con la solución de 2 factores propuesta por Pirkko et al. (1996). Estos autores consideraron aquellos ítems cuyas cargas con el respectivo factor fueran iguales o superiores a 0.50 . Se reportó para el primer factor denominado "Vida" los ítems: 7, 14, 22, 11, 13, 8, 27, 16, 28, 20, 23, 4 y 24. Estos ítems desde el punto de vista de su contenido reflejan el factor Significancia y se refieren en general, a actitudes positivas o negativas ante la vida. Para este factor se reporta una consistencia interna de 0.93 (alfa de Cronbach). El segundo factor denominado por los autores como "Sentimientos", y tiene que ver con el impulso por el control y por la confianza / y desacuerdo en la relación con otros. Se relaciona con aspectos propios de los factores Manejabilidad y Comprensibilidad. Está integrado por los ítems 19, 10, 29, 6, 12, 5, 21, 25. La consistencia interna de este factor fue de 0.81 (alfa de Cronbach).

\section{Cuestionario de actividad física}

Se trata de 5 preguntas, elaboradas por el autor, con base en las cuales se pretende obtener información relacionada con el nivel de actividad física que realizan las personas. Mediante este instrumento se valora no solo el tipo de actividad física que se realiza (además de la implicada en el trabajo y en la casa), sino también, la frecuencia semanal, la duración e intensidad.

\section{Procedimiento}

Se confeccionó un cuadernillo con los instrumentos utilizados en el estudio y luego se procedió a distribuirlo personalmente entre los académicos y administrativos de la Universidad Nacional que laboran en el Campus Presbítero Benjamín Núñez. Este fue distribuido durante el mes de abril de 2008. Se concedió un tiempo de 8 días para que las personas regresaran debidamente llenos los instrumentos, haciendo énfasis en la necesidad de contestar de manera completa cada uno de los cuestionarios. Finalizado el período de espera, se hicieron varias rondas por los distintos espacios laborales de los trabajadores, con el fin de recuperar los instrumentos. Una vez recuperado el $80 \%$ de los cuestionarios entregados, se procedió con el análisis de los datos, haciendo uso del SPSS versión 15.0.

\section{Análisis estadísticos}

Se utilizó estadísticas descriptivas, correlativas e inferenciales. Todos los análisis fueron realizados utilizando el paquete estadístico para las ciencias sociales SPSS versión 15.0. Para todos los análisis se fijó un alfa de $5 \%(\mathrm{p}<0.05)$.

\section{RESULTADOS}

En relación con el ejercicio físico, el análisis de resultados mostró que del total de los encuestados (as), el 68, $1 \%$ realiza algún tipo de actividad física. De manera preferida se menciona la caminata con un $40,6 \%$, seguido por el trabajo con pesas con un $8,7 \%$ y en tercer lugar, practicar deportes de conjunto con un 5,8\%. La frecuencia con que se realiza ejercicio físico por semana se distribuyó de la siguiente forma: Un 11,6 \% lo hace una vez por semana, un 24,6\% lo hace entre 2 y 3 veces por semana y un 31 , $9 \%$ lo hace más de 3 veces por semana. Asimismo, el tiempo dedicado al ejercicio físico se distribuyó como sigue: Un $2,1 \%$ menos de 20 minutos, un $20,3 \%$ lo hace entre 20 y 40 minutos y un $46,4 \%$ lo hace más de 40 minutos. Finalmente, en relación con la intensidad se identificó que, el 7,2\% realiza ejercicio de poca intensidad, el 44,9\% lo hace en un rango de moderada intensidad y el 15,9\% lo realiza con una intensidad alta. 


\section{MHSalud}

URL www.una.ac.cr/mhsalud

En el gráfico 1 se muestra los valores promedio correspondientes a los puntajes registrados en cada uno de los factores de la Calidad de Vida de los trabajadores (as) del Campus Benjamín Núñez.

\section{Gráfico 1: Puntajes promedio en relación con las dimensiones de la calidad de vida}

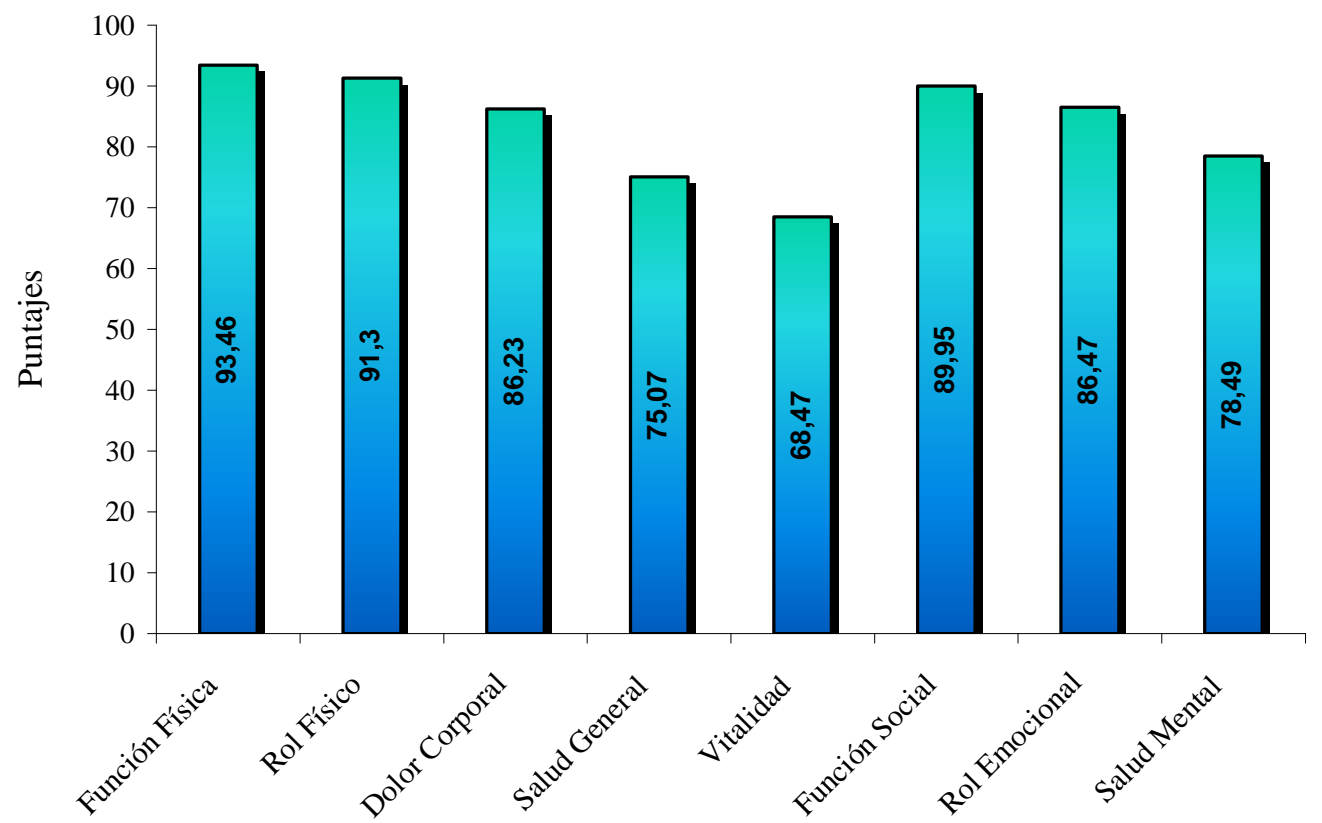

Tal y como se observa en el gráfico anterior, los mayores puntajes se asocian con las dimensiones Función física y Rol físico; por otro lado, que los menores puntajes corresponden a las dimensiones Salud general y Vitalidad. De un máximo de 800 puntos, los encuestados alcanzaron 669,44, lo que representa un $83,68 \%$ del total de puntos posible.

Por otro lado, en cuanto a la valoración de la salud actual comparada con la salud en el pasado reciente (una año atrás), el 8,7\% de los encuestados (as) dijo sentirse mejor ahora que hace un año, el 50, 7 \% opinó tener una salud parecida al año anterior, el $21,7 \%$ manifestó tener una salud algo peor que el año anterior y el 17,4\% expuso sentirse mucho por que hace un año.

El análisis descriptivo de la información relacionada con el Sentido de Coherencia se muestra en el cuadro 1.

Tabla 1. Puntajes promedio correspondientes al Sentido de Coherencia

\begin{tabular}{|c|c|c|c|}
\hline Dimensión & Promedio & Desviación típica & $\begin{array}{l}\text { Puntaje } \\
\text { máximo }\end{array}$ \\
\hline Vida (significancia) & 70,69 & 11,31 & 91 \\
\hline Sentimientos (manejabilidad /comprensibilidad) & 42,08 & 7,32 & 56 \\
\hline Sentido de Coherencia Global & 125 & 18,36 & 154 \\
\hline
\end{tabular}




\section{MHSalud}

URL www.una.ac.cr/mhsalud

En términos proporcionales los puntajes promedio obtenidos en las dimensiones (Vida) y (Sentimientos) es muy similar. Mientras el puntaje promedio de 70, 69 correspondiente a la dimensión Vida, representa un 77, $68 \%$ del puntaje máximo a alcanzar en esa dimensión, el promedio de 42, 08 registrado en la dimensión Sentimientos, representa un 75, $14 \%$ del puntaje máximo alcanzable en la misma.

El cálculo de los percentiles 70, 80 y 90 a partir del puntaje correspondiente a Sentido de Coherencia Global, mostró respectivamente que, el $30 \%$ de los participantes en el estudio obtuvo puntajes arriba de 136, el $20 \%$ registró puntajes por encima de 141 y finalmente, el $10 \%$ alcanzó puntajes por arriba de 147.

Con el propósito de contrastar hipótesis se realizó análisis de varianza de un factor y análisis univariados para valorar interacciones entre factores, así como t-Student para grupos no correlacionados. Se comparó las dimensiones de calidad de vida y sentido de coherencia utilizando como factores: el sexo, la condición laboral, la Unidad Académica de pertenencia, la actividad laboral, la frecuencia del ejercicio, la intensidad y el tiempo. Solo se encontró diferencias significativas en cuanto a la calidad de vida de quienes hacen ejercicio y quienes no lo hacen (cuadro No. 2).

Tabla 2. Calidad de vida según nivel de actividad física

\begin{tabular}{lcccc}
\hline Dimensiones & $\begin{array}{c}\text { Hacen ejercicio } \\
\text { (puntajes promedio) }\end{array}$ & $\begin{array}{c}\text { No hacen ejercicio } \\
\text { (puntajes promedio) }\end{array}$ & t- Student & Significancia \\
\hline Función física & 95,74 & 89,77 & 2,98 & 0,004 \\
Rol físico & 95,74 & 81,81 & 2,22 & 0,03 \\
Dolor corporal & 90,26 & 77,61 & 3,08 & 0.003 \\
Salud general & 79,78 & 65,00 & 3,43 & 0.001 \\
Vitalidad & 72,02 & 60,90 & 2,51 & 0,01 \\
Función social & 93,35 & 82,38 & 2,56 & 0,01 \\
Rol emocional & 90,78 & 77,27 & 1,81 & 0,07 \\
Salud mental & 81,10 & 72,90 & 2,19 & 0,03 \\
\hline
\end{tabular}

Tal y como se observa en el cuadro 2, en todos los casos hay diferencias significativas. Los que hacen ejercicio, comparados con los que no hacen ejercicio, evidencian puntajes promedio significativamente más altos en las dimensiones de la calidad de vida.

En cuanto al factor actividad laboral, se encontró diferencias significativas entre académicos y no académicos en la dimensión "Rol emocional". El puntaje promedio alcanzado por los académicos $(78,37)$ fue significativamente inferior al puntaje promedio alcanzado por los que se dedican a otras actividades $(95,55),(\mathrm{t}=-2,44 ; \mathrm{p}<0.05)$.

La correlación entre Sentido de Coherencia y Calidad de vida arrojó los siguientes resultados (cuadro 3). 
URL www.una.ac.cr/mhsalud

Tabla 3. Matriz de correlaciones entre Calidad de Vida y Sentido de Coherencia

\begin{tabular}{|c|c|c|c|c|c|c|c|c|c|c|}
\hline & $\mathrm{A}$ & B & $\mathrm{C}$ & $\mathrm{D}$ & $\mathrm{E}$ & $\mathrm{F}$ & $\mathrm{G}$ & $\mathrm{H}$ & I & $\mathrm{J} \quad \mathrm{K}$ \\
\hline \multicolumn{11}{|l|}{ Función física (A) } \\
\hline Rol físico (B) & $0,34 * *$ & & & & & & & & & \\
\hline Dolor corporal $(C)$ & $0,35^{* *}$ & $0,60 * *$ & & & & & & & & \\
\hline Vitalidad (E) & $0,41^{*}$ & $0,48 * *$ & $0,57 * *$ & $0,61 * *$ & & & & & & \\
\hline Función social (F) & $0,35 * *$ & $0,75^{* *}$ & $0,60 * *$ & $0,52 * *$ & $0,52 * *$ & & & & & \\
\hline Vida (I) & 0,12 & $-0,18$ & $-0,05$ & $-0,13$ & $-0,07$ & $-0,24 *$ & $-0,11$ & $-0,05$ & & \\
\hline Sentimientos $(\mathrm{J})$ & 0,09 & $0,32 * *$ & $0,34 * *$ & $0,24^{*}$ & 0,22 & $-0,28 *$ & $0,34 * *$ & $0,40 * *$ & 0,14 & \\
\hline Coherencia Global (K) & 0.18 & $0.31 * *$ & 0.21 & $0.28 *$ & $0.26 *$ & $0.34 * *$ & 0.21 & $0.48^{* *}$ & & \\
\hline
\end{tabular}

Todas las dimensiones del factor calidad de vida se correlacionaron de manera significativa entre sí. Sin embargo, entre sentido de coherencia y calidad de vida, solo se obtuvo relaciones significativas entre los factores Vida y Función Social. Asimismo, entre Sentimiento y Rol Físico, Dolor Corporal, Salud General, Función Social, Rol Emocional y Salud mental. Finalmente, el Sentido de Coherencia Global, se correlacionó de manera significativa con los factores: Rol Físico, Salud General, Vitalidad, Función Social y Salud Mental.

\section{DISCUSIÓN}

El propósito principal del presente estudio fue analizar la calidad de vida de académicos y administrativos del Campus Presbítero Benjamín Núñez. Así como también analizar la influencia de los factores Sentido de Coherencia y Ejercicio Físico sobre la Calidad de vida. Los resultados encontrados muestran en términos generales a partir del promedio global, que la calidad de vida del trabajador del Campus Presbitero Benjamín Núñez es “buena”. De acuerdo con Brengelmann (1986) resultados como los encontrados son el reflejo de espacios laborales y de convivencia, donde se cultivan las buenas relaciones sociales y se tiene la posibilidad de desarrollar la propia iniciativa, entre otras condiciones que favorecen el desarrollo de una atmósfera laboral positiva. En términos de Veenhoven (1991) podría hablarse de un nivel de satisfacción vital favorable en los trabajadores (as) del Campus, lo que indudablemente se relaciona con una buena calidad de vida.

Los datos recopilados no evidencian limitaciones importantes en cuanto a la función física y al rol físico de los encuestados. Esto se asocia con la posibilidad general de cumplir con actividades mínimas que garanticen el autocuidado y la capacidad funcional. A pesar de ello, los resultados evidenciaron la presencia de molestias corporales que de alguna manera resultan ser limitantes para la realización de las actividades cotidianas. Asimismo, la valoración personal de la salud, las perspectivas de salud y la resistencia a enfermar, mostraron puntajes sobre los que se puede trabajar para mejorar. Es decir, la posición asumida por los encuestados no es tan optimista como en el caso ideal. Congruente con lo inmediatamente anterior, el sentimiento de energía y vitalidad es el factor más deteriorado en los encuestados. Asimismo, los resultados reportados muestran que la vida social de los encuestados no se ve afectada de manera importante por la salud física o emocional de los mismos. De manera similar, los problemas emocionales que experimenten los encuestados, no parecen ser un gran obstáculo para el desempeño laboral y el logro de rendimiento de los mismos. También el factor salud mental bajo el cual se identifican la tendencia a la depresión, a la ansiedad y bienestar general, fueron aspectos calificados de manera relativamente baja. 


\section{MHSalud}

URL www.una.ac.cr/mhsalud

En cuanto al sentido de coherencia general, el puntaje promedio obtenido muestra un nivel de Sentido de Coherencia relativamente alto, lo que permite suponer una autovaloración suficiente de la competencia psicológica de académicos y administrativos del Campus. Esto puede considerarse según lo propuesto por Antonowsky (1987), Ebert, Tucker y Roth (2002) y Strausser y Lustig (2003), como un factor potenciador de la salud general y de la calidad de vida de los encuestados. A partir de los resultados correspondientes al Sentido de Coherencia, se puede suponer un sentimiento dinámico de confianza en los trabajadores del Campus, lo que permite esperar patrones conductuales flexibles y adaptativos en el comportamiento de los mismos (Antonowsky, 1997).

De manera particular, en el factor "Vida", aunque los resultados mostraron un puntaje promedio que correspondió al 77,68 \% del valor máximo, lo que en criterio de Antonovsky (1997) refiere patrones cognoscitivos optimistas en relación con el contexto inmediato y mediato, también se evidenció la presencia de actitudes negativas ante la vida, lo que por el contrario puede asociarse con estilos cognitivos pesimistas en cuanto al ordenamiento, predicción y explicación de los estímulos internos y externos a que están sujetos los trabajadores (as) del Campus.

Un comportamiento similar se registró para el caso del factor "Sentimientos". El puntaje alcanzado por los encuestados representó un 75,14 \% del máximo, lo que permite inferir una necesidad aceptable de ejercer control y obtener confianza en la relación con otros. Es decir, los resultados promedio registrados en este factor, dejan percibir cierto grado de incertidumbre asociado a circunstancias internas y externas; así como también, cierto nivel de desconfianza en relación con las posibilidades que se tienen para superar las situaciones de la vida presente y futura.

En términos correlativos, el factor "Vida" caracterizado por las actitudes ante la vida, se vinculó negativa y significativamente con la dimensión "Función social" de la calidad de vida. Esto indica que conforme interfieran los problemas de salud física o emocional en la vida social, también aumentan la presencia de actitudes negativas ante la vida. Asimismo, el factor "Sentimientos", caracterizado por el impulso de control y confianza, se correlacionó negativa y significativamente con el factor "Función Social". De manera que, entre menores sean los problemas físicos asociados con las posibilidades de tener vida social, mayor será el sentimiento de control y confianza que se establezca en la relación con otros. Por otro lado, los resultados mostraron que, asociado al sentimiento de control y confianza, existe una mayor capacidad para el desempeño de las tareas cotidianas, menos interferencia de molestias corporales en la ejecución de esas tareas, una valoración positiva de la salud general, estados emocionales adecuados para la vida laboral y una sensación general de bienestar positiva. De manera que, un buen sentido de coherencia especialmente en cuanto al factor "Sentimiento", se asocia de manera importante con la valoración de una buena calidad de vida. En general, estos resultados coinciden con lo expuesto por autores como Lustig (2005), Lundquist (1995), Johansson y otros (1998) y Poppius y otros (1999), quienes destacan el papel que juega el Sentido de Coherencia como variable mediadora en la salud en general y consecuentemente en la calidad de vida.

El contraste de hipótesis evidenció de manera particular relaciones significativas entre la condición "realizar ejercicio físico" y Calidad de vida. Las personas que reportaron hacer ejercicio físico, comparadas con las que no hacen ejercicio, mostraron valorar mejor su condición corporal (más salud física, menos dolor corporal), de manera que sus posibilidades para el desempeño autónomo cotidiano son mayores. Por otro lado, la valoración que hacen las personas que realizan ejercicio físico es mejor que la de los que no hacen ejercicio físico en cuanto al sentimiento de energía y vitalidad, además de mostrar una mejor salud emocional y de una vida social más intensa. 


\section{MHSalud}

URL www.una.ac.cr/mhsalud

Es importante mencionar que la mayoría de los encuestados reportó hacer ejercicio físico. El detalle diferenciado del ejercicio físico que realizan, es consistente con las recomendaciones que brinda el Colegio Americano de Medicina Deportiva (1998), en términos de frecuencia, intensidad y tiempo. Por lo que en ese grupo podría esperarse beneficios significativos en relación con su salud integral. Todo indica que, al hacer ejercicio físico se promociona una mejor salud y consecuentemente una mejor calidad de vida (Ureña, 2005; Hollman y Struder, 2001; Weinberg y Gould, 1999; Comellas y Mecader, 1992; Sánchez, 1996).

En cuanto al Sentido de Coherencia, no se registró diferencias entre las personas que hacen ejercicio físico y las que no lo hacen. Siguiendo a Antonowsky (1987), podría decirse que ambos grupos de personas tienen una posición básica similar ante la vida. Es decir, el hecho de hacer o no hacer ejercicio físico, parece no tener relación con la visión de vida de los académicos y administrativos del Campus.

Finalmente, también se encontró diferencias significativas entre quienes ejercen funciones meramente académicas y quienes cumplen con otro tipo de actividades. En el caso de los primeros, pareciera que los problemas emocionales tienden a interferir con más fuerza en el desempeño y esmero laboral.

Como conclusión general puede afirmarse que la valoración de la calidad de vida que hacen los académicos y administrativos del Campus Presbítero Benjamín Núñez es "buena", aunque no la mejor. Esto significa que debería invertirse en propuestas que fortalezcan la salud en general y la Calidad de vida de estos trabajadores (as). Por otro lado, en relación con el Sentido de Coherencia, es preciso también invertir en acciones que fortalezcan la posición de los trabajadores (as) del Campus en cuanto a su actitud ante la vida; sobre todo reforzando el sentido de confianza en las propias potencialidades y la capacidad adaptativa. En este sentido, el ejercicio físico mostró ser una opción importante para contribuir con la calidad de vida de los trabajadores del Campus y perfila convertirse en un bastión fundamental en la constitución de lo que Peterken (1996) denomina "Universidades Saludables".

\section{REFERENCIAS}

Alonso, J. Prieto, L. y Antó, J. M. (1995). La versión española del SF-36 Health Survey (Cuestionario de Salud SF-36): un instrumento para la medida de los resultados clínicos. Medicina Clínica, (104), 20, 771-776.

American College of Sport Medicine (1998). Guidelines for Exercise Testing and Prescription. Baltimore: Lippincott Williams and Wilkins.

Antonovsky, A. (1996). The salutogenic model as a theory to guide health promotion. Health Pro motion International, 11, 11-18.

Antonowsky, A. (1997). Salutogenese . Zur Entmystifizierung der Gesundheit. Tübingen: Dgvt.

Antonowsky, A. (1987). Unraveling the Mystery of Health: How People Manage Stress and Stay Well. San Francisco: Jossey-Bass.

Baker, M. North, D. y Smith, D. F. (199 7). Burnout, sense of coherence and sources of salutogenesis in social workers. Psychology: A Journal of Human Behavior, 34, 22 -26. 


\section{MHSalud}

URL www.una.ac.cr/mhsalud

Brengelmann, J.C. (1986). Stress, superación y calidad de vida en personas sanas y enfermas. Evaluación Psicológica, 2, 47-77.

Carmel, S. Anson, O., Levenson, A., Bonneh, D. y Mahoz, B. (1991). Life events, sense of coherence and health: gender differences on the kibbutz. Social Science and Medicine, 32, 1089-1096.

Comellas, M. J. y Mercader, I. (1992). Finalidades educativas del deporte en educación secundaria. Apunts, 29, 32-43.

Diener, E. (1994). Assessing subjective well-being: Progress and opportunities. Social Indicators Research, 31, 103-157.

Evans, D. R. (2005). Health promotion: Enhancing the quality of life population at large. En D. M. Rommey y R. I. Brown (Eds.), Improving the Quality of Life in Normal and Disabled Populations. Dordrecht: Kluver Academic Publisher.

Ebert, S., Tucker, D., \& Roth, D. (2002). Psychological resistence factors as predictors of general health status and physical symptom reporting. Health and Medicine, 7, 363-375.

Fernández, L. y García, M. (1999). Psicología preventiva y calidad de vida. En M. A. Simón (Ed.), Manual de Psicología de la Salud. Fundamentos, Metodología y Aplicaciones (págs. 115 -155). Madrid: Biblioteca Nueva.

Flannery, R. B. y Flannery, G. J. (1990). Sense of coherence, life stress, and psychological distress: a prospective methodological inquiry. Journal of Clinical Psychology, 46, 415-420.

Gilbar, O. (1998). Relationship between burnout and sense of coherence in health social workers. Social Work in Health Care, 26 , 39-49.

Godoy, J. F. (1999). Psicología de la Salud: Delimitación conceptual. En M. A. Simón (Ed.), Manual de Psicología de la Salud. Fundamentos, Metodología y Aplicaciones (págs. 39-70 ). Madrid: Biblioteca Nueva.

Gracia, E., Herrero, J. y Musito, G. (1995). El apoyo social. Barcelona: PPU.

Halpern, A. S. (1994). Quality of life for students with disabilities in transitions from school to adulthood. Social Indicators Research, 33 , 193 -236.

Hollmann, W., y Struder, H. (2001). Brain, Psyche, Mind and Muscular Activity. En W. Hollmann; D. Kurz y J. Mester (Eds.), Current Result on Health and Physical Activity (págs. 87-113). Stuttgart: Schattauer.

Johansson, I. Larsson, G. Hamrin, E. (1998). Sense of coherence, quality of life, a function among elderly hip fracture. Aging, 10, 3 77-384.

LaForge, R. (1995). Exercise associated mood alteratios: a review of interactive neurobiologic mechanisms. Medicine Exercise Nutrition and Health, 4 (1), 17-32.

Lundquist, T. (1995). Chronic cannabis use and the sense of coherence. Life Science, 56 , 2145 -2150. 


\section{MHSalud}

URL www.una.ac.cr/mhsalud

Levy, L. y Anderson, L. (1980). La tensión psicosocial. Población, Ambiente y calidad de vida. México: El Manual Moderno.

Lustig, D. (2005). The Adjustment Process for Individuals With Spinals Cord Injury: The effect of perceived premorbid sense of coherence. Rehabilitation Counseling Bulletin, 48(3), 146 -156.

Lustig, D., Rosenthal, D., Strausser, D. \& Haynes, K. (2000). The relationship of sense of coherence to life satisfaction for students with disabilities. Rehabilitation Counseling Bulletin, 43, 134-141.

McTeer, W. y Curtis, J. (1993). Sport and physical activity and subjective well being: national panel data for the U.S. International Review for the Sociology of Sport, 28 (4), 397-414.

Moreno, B. González, J. L. y Garrosa, E. (1999). Burnout docente, sentido de coherencia y salud percibida. Revista de Psicopatología Clínica (4), 3, 163-180.

Moreno-Jiménez, B., Alonso, M., y Álvarez, E. (1997). Sentido de coherencia, Personalidad Resistente, autoestima y salud. Revista de Psicología de la Salud, 9, 115 -138.

Morgan, W. P. y Goldston, S.E. (1987). Exercise and mental health. New York: Hemisphere.

Nordenfelt, L. (1993). Quality of life, Health and Happiness. Aldershot: Avebury.

Peterken, D., Steinmetz, B., Renwick, R., Rootman, I., Brown, I., Sehdey, H., Phillips, S. y Smith, T. (1999). The community Quality of Life Project: a health promotion approach to understanding communities. Health Promotion International, 14, 197-21 0.

Pirkko, S. , Matti, H., Erkki, K y Veikko, A. (1996). Comparing two self report measures of coping The sense of coherence scale and the defense style questionnaire. Journal of Clinical Psychology, $52(5), 517-524$.

Plante, T.G. y Rodin, J. (1990). Physical fitness and enhanced psychological health. Current Psychology: Research and Reviews, 9, 3 -24.

Palsson, M.B., Hallberg, I.R., Norberg, A. y Bjoervell, H. (19 96). Burnout, empathy and sense of coherence among Swedish district nurses before and alter systematic clinical supervision. Scandinavian Journal of Caring Sciences, 10, 19 -26.

Poppius, E. Tenkanen, L. Kalimo, R. \& Heinsalmi, P. (1999). The sense of coherence, occupation and the risk of coronary heart disease in the Helsinski Heart Study. Social Science Medicine, 49, 109 -120 .

Ryland, E. y Greenfeld, S. (1991). Work, stress and well-being: an investigation of Antonowskys Sense of Coherence Model. En P.L. Perrewe (Ed.), Handbook of job stress (special issue). Journal of Social Behavior and Personality, 6, 39-54.

Rommey, D.M., Brown, R.I., y Fry, P.S. (19 94). Improving the quality of life: Prescriptions for change. Social Indicators Research, 33, 237-272. 


\section{MHSalud}

URL www.una.ac.cr/mhsalud

Robinson, N.S. y Garber, J. (1995). Social support and psychopathology across the life span. En D. Cicchetti y D. J. Cohen (Eds.), Developmental Psychopathology. Risk, Disorder and Adaptation. New York: John Wiley.

Sánchez, F. (1996). La actividad física orientad a hacia la salud. Madrid: Biblioteca Nueva.

Steptoe, A., Moses, J., Edwards, S. y Mathews, A. (1993). Exercise and responsibility to mental stress: discrepancies between the subjective and physiological effects of aerobic training. International Journal of Sport Psychology, 24 (2), 110-129.

Stephens, T. (1988). Physical activity and mental health in the United States and Canada: Evidence from four population survey. Preventive Medicine, 17, 35-47.

Schalock, R.L. (1994). Quality of life, quality enhancement, and quality assurance: Implications for program planning and evaluation in the field of mental retardation and develop mental disabilities. Evaluation and Program Planning, 17, 121 -131.

Simón, M.A. (1999). Manual de Psicología de la Salud. Fundamentos, Metodología y Aplicaciones. Madrid: Biblioteca Nueva.

Strausser, D., \& Lustig, D. (2 003). The moderating effects of sense of coherence on work adjustment. Journal of Employment Counseling, 40, 129-140.

Ureña, P. (2005). Correlatos psicológicos y actividades deportivas. Universidad Nacional, Heredia: Programa de publicaciones.

Veenhoven, R. (1991). Questions on Happiness: Classical topics, modern answers, blind spots. En F. Strack, M. Argyle y N. Schwars (Eds), Subjective Well - Being. An Interdisciplinary Perspective. Oxford: Pergamon Press.

Vilagut, G., Ferrer, M., Rajmil, L., Rebollo, P., Permanyer-Miralda, G., Santd, R., Valderas, J.,m Riber, A., Domingo-Salvany, A. y Alor, J. (2005). El cuestionario de salud SF-36: una década de experiencia y nuevos desarrollos. Gaceta Sanitaria (1-16), 12, Marzo-Abril, No. 2.

Ware, J.E. y Sherbourne C.D. (1992). The MOS 36 - item short form health survey (SF-36). I. Med Care, 39, 473-483.

Weinberg, R. y Gould, D. (1999). Foundations of sport and exercise psychology. Champaign, Il: Human Kinetics.

Weyerer, S. y Kupfer, B. (1994). Physical exercise and psychological health. Sports Medicine, 17 (2), 108-116.

Fecha de recepción: 07 de agosto del 2008

Fecha de aceptación: 10 de octubre del 2008

Fecha de publicación: 31 de diciembre del 2008 